\title{
Treatment of Intractable Midbrain Tremor with a Pedinculopontine Nucleus Radiofrequency Lesion
}

\author{
Ali AKAY'1, Mete RUKSEN ${ }^{1}$, Burhanettin ULUDAG ${ }^{2}$, Sertac ISLEKEL ${ }^{1}$ \\ ${ }^{1}$ Kent Hospital, Department of Neurosurgery, Izmir, Turkey \\ ${ }^{2}$ Ege University, Faculty of Medicine, Department of Neurology, Izmir, Turkey \\ This study has been presented at the $33^{\text {rd }}$ Annual Scientific Congress of Turkish Neurosurgical Society 2019 at Antalya, Turkey
}

Corresponding author: Ali AKAY dr.aliakay@gmail.com

\section{ABSTRACT}

In this report, we present the case of a 21 -year-old female with midbrain tremor involving atypical features, treated using a pedinculopontine nucleus lesion. Different targets for the treatment of midbrain tremor using radiofrequency lesion have been reported. In this case, stimulation of the thalamic ventralis intermedius nucleus and subthalamic nucleus produced no satisfactory improvement in tremor frequency and amplitude. A third goal was to obtain a significant response in terms of these outcomes with pedinculopontine nucleus stimulation.

According to the literature, this is the first case of midbrain tremor treated with a pedinculopontine nucleus radiofrequency thermocoagulation lesion.

KEYWORDS: Midbrain tremor, Pedinculopontine nucleus, Radiofrequency thermocoagulation lesion

ABBREVIATIONS: MT: Midbrain tremor, Vim: ventralis intermedius nucleus, PPN: pedinculopontine nucleus, Rf-Tc: Radiofrequency thermocoagulation, MRI: Magnetic resonance imaging, PPNc: Pars compacta, PPNd: Pars dissipates

\section{INTRODUCTION}

$\mathrm{M}$ idbrain tremor (MT), which is also known as rubral, thalamic, mesencephalic or Holmes tremor, is a rare type of hyperkinetic movement disorder. It was described by Holmes in 1904 and manifests as a combination of resting, postural and kinetic tremors (7). The amplitude of tremor at rest is low but can increase to an uncontrollable intensity, depending on the posture and movement (2). The exact pathophysiology of MT is unknown; however, damage to dopminergic nigrostriatal, cerebello-thalamo-cortical and dentato-rubro-olivary fibres may explain symptoms at rest $(3,13)$. Radiological examinations of patients with MT revealed both normal and abnormal findings (4). MT usually develops secondary to lesions located in the thalamus, upper brainstem and cerebellum. Lesions associated with MT may have different aetiologies, such as ischaemia, haemorrhagic cerebrovascular disorders, haemorrhage secondary to vascular malformations, head trauma, tumours, demyelination and infection (3). Many drugs, including benzodiazepines, propranolol, anticholinergics, bromocriptine and levodopa, have been used to treat MT (1).

In patients with drug-resistant MT, treatment using radiofrequency lesion of the thalamic ventralis intermedius nucleus (Vim) has shown satisfactory results; however, better results have been reported using deep brain stimulation (8). In this report, treatment of MT with atypical features using radiofrequency thermocoagulation ( $\mathrm{Rf}-\mathrm{Tc}$ ) lesion of a PPN is presented.

\begin{tabular}{llll}
\hline Ali AKAY & (D) : 0000-0002-7187-398X & Burhanettin ULUDAG (1) : 0000-0001-5023-4321 \\
Mete RUKSEN (1) : 0000-0002-0521-8143 & Sertac ISLEKEL & (D) : 0000-0001-8091-1426
\end{tabular} 


\section{CASE REPORT}

A 21-year-old female patient was diagnosed with right MT 7 months following her initial presentation. A $7.5 \mathrm{~Hz}$-frequency constant rhythm oscillation was detected at rest and with movement. Cranial magnetic resonance imaging (MRI) revealed no radiopathological findings. Medical treatment, including appropriate doses of beta-adrenergic receptor blocking, anti-epileptic (primidone, gabapentin and topiramate) and anticholinergic (biperidene) agents, produced no response. The patient was referred to our clinic for radiofrequency lesion of the nucleus. Patients informed consent was obtained according to the Declaration of Helsinki.The left thalamic Vim, subthalamic nucleus and PPN were selected as targets. T1 and T2 weighted MRI were performed using a Leksell stereotactic frame. Coordinates for the three separate targets were determined using the Leksell GammaPlan ${ }^{\circledR}$ program on T1-weighted MRI in the light of the relevant literature $(4,13,14)$. In addition, T2-weigted MR images were used for STN targeting. Initially, thalamic Vim stimulation was performed at a frequency of $100-130 \mathrm{~Hz}$ and voltage of 1-3; however, the response was limited. Using these same parameters applied to subthalamic nucleus stimulation, a partial response was observed in the amplitude of the tremor, but the frequency did not improve. When stimulation at 2 volts with a frequency of $100 \mathrm{~Hz}$ was applied to the left PPN coordinates, a dramatic response in tremor amplitude and frequency was obtained, with contraction of the right upper and lower extremities. A single lesion was applied for 60 seconds at $70^{\circ} \mathrm{C}$ to PPN. Two different trajectories were used for the Vim and the STN stimulation. The PPN stimulation was performed from the same trajectory used for the STN stimulation.

\section{RESULTS}

Following this procedure, MT was completely controlled. Left hemiparesis and ataxia were observed on the right side but were resolved within 1 week. After 6 months, the tremor remained completely controlled and neurologically stable. The patient consented to publication of her image Postoperative 1st month T2 weighted MRI shows the distance of the anatomical borders to the lesion (Figure 1).

\section{DISCUSSION}

MT is typically characterised by flexion-extension oscillation at rest, which disappears during sleep. However, with movement, the tremor frequency may increase. This condition has a greater effect on the proximal muscles of the unilateral upper extremities than on the distal muscles. Hemiparesis and cranial nerve disorders resulting from midbrain injury are observed with radiopathological lesions. The frequency of the presented case was constant at rest and measured $7.5 \mathrm{~Hz}$ with posture and movement. Oscillation affected the right distal muscle groups more than the proximal muscles. There were no abnormal radiological findings based on MRI. In this case of MT with atypical features, radiofrequency stimulation of the thalamic Vim and subthalamic nucleus resulted in a partial reduction in amplitude intensity, but there were no other changes. Therefore, PPN was selected as a third target. Deep brain stimulation of PPN has demonstrated improvements in freezing and gait disorders associated with Parkinson's disease (8). However, to date, there have been no studies on the association between MT and PPN stimulation. In this case study, levodopa resistance and a lack of response to medical treatment led the surgical and neurology teams to recommend PPN stimulation. After informing the patient about PPN stimulation and receiving her approval, PPN was included among the targets for stimulation.

PPN is located on the dorsolateral side of the pontomesencephalic tegmentum at the upper portion of the pons. It is a part of the mesencephalic locomotor region in the upper brain stem (14). PPN is composed of two sub-cores, called the pars compacta (PPNc) and pars dissipatus (PPNd) (5). PPN has afferent and efferent projections, with both cholinergic and non-cholinergic neurons to the cerebral cortex, thalamus, basal ganglia, cerebellum and spinal cord. PPNc comprises cholinergic neurons along a large part of the superior cerebellar peduncle border (10). PPNd is composed of glutamatergic neurons and the remaining cholinergic neurons, most of which are located at the mid-encephalon and mid-pons levels along the dorsolateral pons (10). PPNc and PPNd also contain GABAergic inhibitory neurons (11). PPN receives projections from the cerebral cortex through the basal ganglia and extrapyramidal system, including those from the patterned nets, motor cortex, face, arm and trunk and leg re-

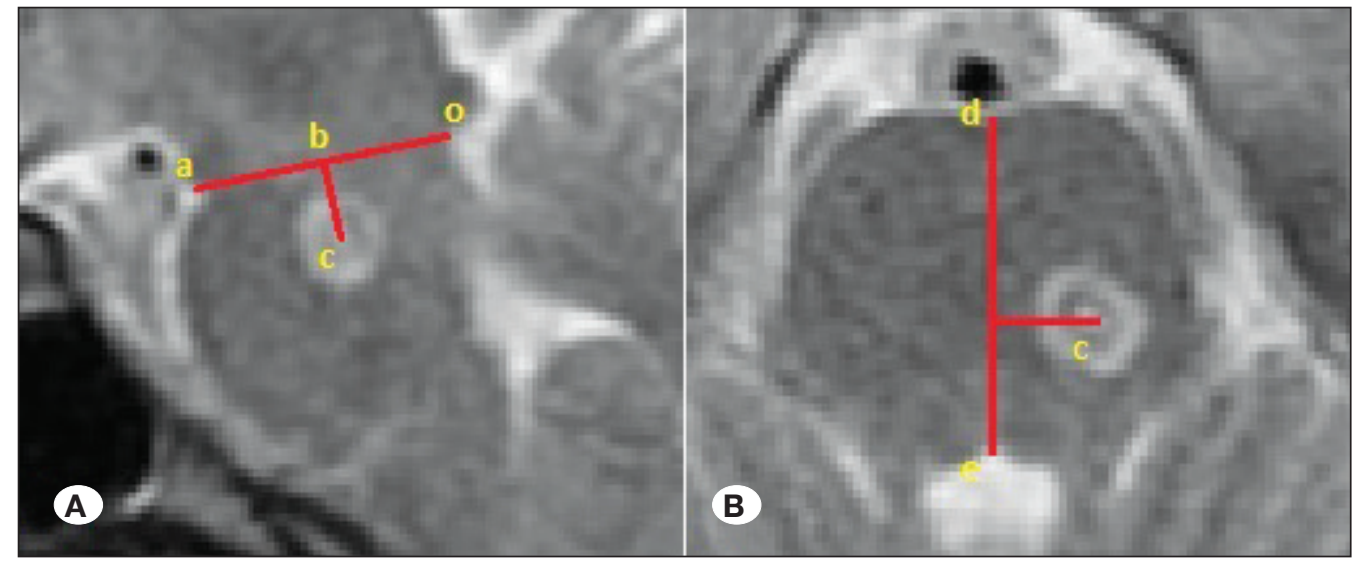

Figure 1: A) Postoperative sagittal T2 weight -MRI. (ao) pontomesencefalic junction (PMJ) anterior-posterior lenght: $20.5 \mathrm{~mm}$, (bc): PMJ and center of Rf-Tc lesion length: $4.86 \mathrm{~mm}$. B) Postoperative axial T2 weight-MRI. (de) anteriorposterior brain stem midline length: 22,2 mm, (de-c) anterior-posterior brain stem midline and center of Rf-Tc lesion length: $7,39 \mathrm{~mm}$. 
gions, in particular Brodmann area 4. PPNc is a component of the feedback loop between the thalamus, limbic system and spinal cord and of the ascending reticular activating system, in which cortical stimulation is modulated by rising cholinergic connections to the thalamus (6). In the treatment of MT with thalamic Vim stimulation, a 57\%-90\% improvement in tremor ratings was reported (12). Positive results have been observed following dual stimulation of the Vim-subthalamic, ventral oralis anterior-ventral oralis posterior and Vim-globus pallidus nuclei (12). Compared with other types of tremors, the need for higher-voltage stimulation in MT has been reported (9). The success of high-voltage and dual stimulation, in which a larger area is affected, suggests multiple pathways in the pathophysiology of MT.

\section{CONCLUSION}

In this case study, complex MT treatment was achieved using Rf-Tc lesion of PPN. Review of the literature suggests that this is the first case of MT treated in this way. Our findings prompt us to consider whether PPN is a new target for intractable midbrain tremor.

\section{REFERENCES}

1. Alqwaifly M: Treatment responsive Holmes tremor: Case report and literature review. Int J Health Sci (Qassim) 10(4):558-562, 2016

2. Bala VM: Uncommon forms of tremor. In: Watts RL, Koller WC (eds), Movement Disorder. Neurologic Principles and Practice. New York: McGraw-Hill, 1997:391-392

3. Defer GL, Remy P, Malapert D, Ricolfi F, Samson Y, Degos JDI: Rest tremor and extrapyramidal symptoms after midbrain haemorrhage: Clinical and 18F-dopa PET evaluation. J Neurol Neurosurg Psychiatry 57:987-989, 1994

4. Deuschl G, Bain P, Brin M: Consensus statement of the Movement Disorder Society on tremor. Ad hoc Scientific Committee. Mov Disord 13(3):2-23, 1998

5. Goetz L, Bhattacharjee M, Ferraye MU, Fraix V, Maineri C, Nosko D, Fenoy AJ, Piallat B, Torres N, Krainik A, Seigneuret E, David O, Parent M, Parent A, Pollak P, Benabid AL, Debu B, Chabardès S: Deep brain stimulation of the pedunculopontine nucleus area in Parkinson disease: MRI-based anatomoclinical correlations and optimal target. Neurosurgery 84:2:506-518, 2019
6. Grabli D, Karachi C, Folgoas E, Monfort M, Tande D, Clark S, Civelli O, Hirsch EC Françoiset C: Gait disorders in parkinsonian monkeys with pedunculopontine nucleus lesions: A tale of two systems. J Neurosci 33:11986-11993, 2013

7. Holmes G: On certain tremors in organic cerebral lesion. Brain 27:327-375,1904

8. Khan SA, Javed S, Mooney L,White P, Plaha P, Whone A, Gill SS: Clinical outcomes from bilateral versus unilateral stimulation of the pedunculopontine nucleus with and without concomitant caudal zona incerta region stimulation in Parkinson's disease. Br J Neurosurg 26:722-725, 2012

9. Lavoie B, Parent A: Pedunculopontine nucleus in the squirrel monkey: Projections to the basal ganglia as revealed by anterograde tract-tracing methods. J Comp Neurol 344:210231, 1994

10. Lavoie B, Parent A: Pedunculopontine nucleus in the squirrel monkey: Cholinergic and glutamatergic projections to the substantia nigra. J Comp Neurol 344:232-241,1994

11. Mesulam M, Geula C, Bothwell MA, Hersh LB: Human reticular formation: Cholinergic neurons of the pedunculopontine and laterodorsal tegmental nuclei and some cytochemical comparisons to forebrain cholinergic neurons. J Comp Neurol 283:611-633, 1989

12. Ramirez-Zamora A, Okun MS: Deep brain stimulation for the treatment of uncommon tremor syndromes. Expert Rev Neurother 16(8):983-997, 2016

13. Rye DB, Saper CB: Pedunculopontine tegmental nucleus of the rat: Cytoarchitecture, cytochemistry, and some extrapyramidal connections of the mesopontine tegmentum. J Comp Neurol 259:483-528,1987

14. Thevathasan W, Debu B, Aziz T, Bloem BR, Blahak C, Butson C, Czernecki V, Foltynie T, Fraix V, Grabli D, Joint C, Lozano AM, Okun MS, Ostrem J,Pavese N, Schrader C, Tai CH, Krauss JK, Moro E: Pedunculopontine nucleus deep brain stimulation in Parkinson's disease: A clinical review. Mov Disord 33:10-20, 2018 